\title{
Classes of harmonic functions defined by convolution
}

\section{Jacek Dziok ${ }^{1}$}

Received: 25 March 2019 / Accepted: 13 September 2019 / Published online: 11 October 2019

(c) The Author(s) 2019

\section{Abstract}

The object of the present paper is to investigate classes of harmonic functions defined by convolution. Some necessary and sufficient conditions, topological properties, radii of convexity and starlikeness, as well as extreme points for the classes are considered.

Keywords Harmonic functions · Convolution - Subordination · Extreme points · Starlike functions

Mathematics Subject Classification Primary 30C55 · Secondary 30C45

\section{Introduction}

Let $\mathcal{H}$ denote the class of complex harmonic functions in $\mathbb{U}:=\mathbb{U}(1)$, where $\mathbb{U}(r):=$ $\{z \in \mathbb{C}:|z|<r\}$, and let $\mathcal{H}_{0}$ denote the class of functions $f \in \mathcal{H}$ of the form

$$
f(z)=z+\sum_{n=2}^{\infty}\left(a_{n} z^{n}+\overline{b_{n} z^{n}}\right) \quad(z \in \mathbb{U}) .
$$

By $\mathcal{S}_{\mathcal{H}}$ we denote the class of functions $f \in \mathcal{H}_{0}$, which are univalent and sense preserving in $\mathbb{U}$.

We say that a function $f \in \mathcal{S}_{\mathcal{H}}$ is harmonic starlike in $\mathbb{U}(r)$ if $f$ maps the circle $\partial \mathbb{U}(r)$ onto a closed curve that is starlike with respect to the origin, i.e., $\frac{\partial}{\partial t}\left(\arg f\left(r e^{i t}\right)\right)>0 \quad(0 \leq t \leq 2 \pi)$ or equivalently

$$
\operatorname{Re} \frac{D_{\mathcal{H}} f(z)}{f(z)}>0 \quad(|z|=r)
$$

Jacek Dziok

jdziok@ur.edu.pl

1 Faculty of Mathematics and Natural Sciences, University of Rzeszów, ul. Prof. Pigonia 1, 35-310 Rzeszów, Poland 
where

$$
D_{\mathcal{H}} f(z):=z f_{z}^{\prime}(z)-\bar{z} f_{\bar{z}}^{\prime}(z) \quad(z \in \mathbb{U}) .
$$

Let us consider the function $\varphi \in \mathcal{H}$ of the form

$$
\varphi=u+\bar{v}, \quad u(z)=z+\sum_{n=0}^{\infty} u_{n} z^{n}, \quad v(z)=\sum_{n=0}^{\infty} v_{n} z^{n} \quad(z \in \mathbb{U})
$$

with

$$
\left|u_{n}\right| \geq 1,\left|v_{n}\right| \geq 1 \quad(n=2,3, \ldots) .
$$

We say that a function $f \in \mathcal{H}$ of the form (1) is correlated with the function $\varphi$, if

$$
u_{n} a_{n}=-\left|u_{n}\right|\left|a_{n}\right|, v_{n} b_{n}=\left|v_{n}\right|\left|b_{n}\right| \quad(n=2,3, \ldots) .
$$

Let $|\eta|=1$. We say that a function $f \in \mathcal{H}$ of the form (1) is $\eta$-correlated with the function $\varphi$, if it is correlated with the function

$$
\varphi_{\eta}(z):=\eta^{-1} \varphi(\eta z) \quad(z \in \mathbb{U}) .
$$

In particular, functions correlated with the function

$$
\varphi(z)=2 \operatorname{Re} \frac{z}{1-z}=\sum_{n=1}^{\infty}\left(z^{n}+\bar{z}^{n}\right) \quad(z \in \mathbb{U}),
$$

are the functions with negative coefficients introduced by Silverman [34]. Also, functions $\eta$-correlated with the function $\varphi$ of the form (4) are the functions with varying coefficients defined by Jahangiri and Silverman [21] (see also [17]). These functions are intensively investigated by many authors (for example, see [6-23,27-34]).

We say that a function $f: \mathbb{U} \rightarrow \mathbb{C}$ is subordinate to a function $F: \mathbb{U} \rightarrow \mathbb{C}$, and write $f(z) \prec F(z)$ (or simply $f \prec F$ ), if there exists a complex-valued function $\omega$ which maps $\mathbb{U}$ into oneself with $\omega(0)=0$, such that

$$
f(z)=F(\omega(z)) \quad(z \in \mathbb{U}) .
$$

In particular, if $F$ is univalent in $\mathbb{U}$, we have the following equivalence:

$$
f(z) \prec F(z) \Longleftrightarrow[f(0)=F(0) \quad \text { and } \quad f(\mathbb{U}) \subset F(\mathbb{U})]
$$

Let $A, B$ be real parameters with $B>\max \{0, A\}$ and let $\varphi$ be given by (2). We denote by $\mathcal{W}_{\mathcal{H}}(\varphi ; A, B)$ the class of functions $f \in \mathcal{S}_{\mathcal{H}}$ such that

$$
\frac{D_{\mathcal{H}}(\varphi * f)}{\varphi * f} \prec \frac{1+A z}{1+B z}
$$


where $f * \varphi$ denote the convolution of $f$ and $\varphi$, defined by

$$
(\varphi * f)(z)=\sum_{n=0}^{\infty} a_{n} u_{n} z^{n}+\sum_{n=1}^{\infty}{\overline{b_{n} v_{n}}}_{\bar{z}}^{n} \quad(z \in \mathbb{U})
$$

Also, by $\mathcal{V}_{\mathcal{H}}(\varphi ; A, B)$ we denote the class of functions $f \in \mathcal{S}_{\mathcal{H}}$ such that

$$
\frac{(\varphi * f)(z)}{z} \prec \frac{1+A z}{1+B z} .
$$

Let $\mathcal{T}^{\eta}(\varphi)$ denote the class of functions $f \in \mathcal{H}$ of coefficients $\eta$-correlated with the function $\varphi$, i.e.,

$$
u_{n} a_{n}=-\left|u_{n}\right|\left|a_{n}\right| \eta^{1-n}, \overline{v_{n} b_{n}}=\left|v_{n}\right|\left|b_{n}\right| \eta^{1+n} \quad(n=2,3, \ldots) .
$$

Moreover, let us define

$$
\begin{aligned}
\mathcal{W}_{\mathcal{H}}^{\eta}(\varphi ; A, B) & :=\mathcal{T}^{\eta}(\varphi) \cap \mathcal{W}_{\mathcal{H}}(\varphi ; A, B), \quad \mathcal{V}_{\mathcal{H}}^{\eta}(\varphi ; A, B):=\mathcal{T}^{\eta}(\varphi) \cap \mathcal{V}_{\mathcal{H}}(\varphi ; A, B), \\
\mathcal{W}_{\mathcal{H}}^{\eta}(\varphi ; \alpha) & :=\mathcal{W}_{\mathcal{H}}^{\eta}(\varphi ; 2 \alpha-1, B), \quad \mathcal{W}_{\mathcal{H}}^{\eta}(\varphi ; \alpha):=\mathcal{W}_{\mathcal{H}}^{\eta}(\varphi ; 2 \alpha-1, B) .
\end{aligned}
$$

In the paper, we obtain some analytic criteria, radii of convexity and starlikeness for defined classes of functions. Using extreme points theory, we obtain coefficients estimates, distortion theorems and integral mean inequalities. Some applications of the obtained results are also considered.

\section{Analytic criteria}

Let $\mathcal{V} \subset \mathcal{H}, \mathbb{U}_{0}:=\mathbb{U} \backslash\{0\}$. Motivated by Ruscheweyh [31] we define the dual set of $\mathcal{V}$ by

$$
\mathcal{V}^{*}:=\left\{f \in \mathcal{S}_{\mathcal{H}}: \bigwedge_{q \in \mathcal{V}}(f * q)(z) \neq 0 \quad\left(z \in \mathbb{U}_{0}\right)\right\}
$$

First, we show that the defined classes of functions can be presented as dual sets.

\section{Theorem 1}

$$
\mathcal{W}_{\mathcal{H}}(\varphi ; A, B)=\left\{\varphi * \psi_{\xi}:|\xi|=1\right\}^{*}
$$

where

$$
\psi_{\xi}(z):=z \frac{(B-A) \xi+(1+A \xi) z}{(1-z)^{2}}-\bar{z} \frac{2+(A+B) \xi-(1+A \xi) \bar{z}}{(1-\bar{z})^{2}} \quad(z \in \mathbb{U})
$$

Proof Let $f \in \mathcal{S}_{\mathcal{H}}$. Then $f \in \mathcal{W}_{\mathcal{H}}(\varphi ; A, B)$ if and only if it satisfies (5) or equivalently

$$
\frac{D_{\mathcal{H}}(\varphi * f)}{\varphi * f} \neq \frac{1+A \xi}{1+B \xi} \quad(\xi \in \mathbb{C},|\xi|=1)
$$


Since $f=h+\bar{g}$ for some analytic functions $h, g$, and

$$
D_{\mathcal{H}} h(z)=h(z) * \frac{z}{(1-z)^{2}}, \quad h(z)=h(z) * \frac{z}{1-z},
$$

we have

$$
\begin{aligned}
& 0 \neq(1+B \xi) D_{\mathcal{H}}(\varphi * f)(z)-(1+A \xi)(\varphi * f)(z) \\
& =(1+B \xi) D_{\mathcal{H}}(h * u)(z)-(1+A \xi)(h * u)(z) \\
& -\left[(1+B \xi) \overline{D_{\mathcal{H}}(g * v)(z)}+(1+A \xi) \overline{(g * v)(z)}\right] \\
& =(h * u)(z) *\left(\frac{(1+B \xi) z}{(1-z)^{2}}-\frac{(1+A \xi) z}{1-z}\right) \\
& -\overline{(g * v)(z)} *\left(\frac{(1+B \xi) \bar{z}}{(1-\bar{z})^{2}}+\frac{(1+A \xi) \bar{z}}{1-\bar{z}}\right) \\
& =f(z) *\left(\varphi * \psi_{\xi}\right)(z)
\end{aligned}
$$

Thus, $f \in \mathcal{W}_{\mathcal{H}}(\varphi ; A, B)$ if and only if $f(z) *\left(\varphi * \psi_{\xi}\right)(z) \neq 0$ for $z \in \mathbb{U}_{0}, \quad|\xi|=1$, i.e., $\mathcal{W}_{\mathcal{H}}(\varphi ; A, B)=\left\{\varphi * \psi_{\xi}:|\xi|=1\right\}^{*}$.

Similarly as Theorem 1 we prove the following theorem.

\section{Theorem 2}

$$
\mathcal{V}_{\mathcal{H}}(\varphi ; A, B)=\left\{\varphi * \delta_{\xi}:|\xi|=1\right\}^{*}
$$

where

$$
\delta_{\xi}(z):=z \frac{(B-A) \xi+(1+A \xi) z}{1-z}+\bar{z} \frac{1+B \xi}{1-\bar{z}} \quad(z \in \mathbb{U}) .
$$

Theorem 3 Let $f \in \mathcal{H}$ be of the form (1) and

$$
\sum_{n=2}^{\infty}\left(\left|\gamma_{n}\right|\left|a_{n}\right|+\left|\delta_{n}\right|\left|b_{n}\right|\right) \leq B-A
$$

where

$$
\gamma_{n}=u_{n}(n(1+B)-(1+A)), \quad \delta_{n}=v_{n}(n(1+B)+(1+A)) .
$$

Then $f \in \mathcal{W}_{\mathcal{H}}(\varphi ; A, B)$.

Proof Let $f \in \mathcal{H}$ be a function of the form (1). By a theorem of Lewy [25], a necessary and sufficient condition for $f$ to be locally univalent and sense preserving in $\mathbb{U}$ is that

$$
\left|h^{\prime}(z)\right|>\left|g^{\prime}(z)\right| \quad(z \in \mathbb{U}) .
$$

By (3) we obtain

$$
\frac{\left|\gamma_{n}\right|}{B-A} \geq n, \frac{\left|\delta_{n}\right|}{B-A} \geq n \quad(n=2,3, \ldots)
$$


and, by (11) we get

$$
\sum_{n=2}^{\infty}\left(n\left|a_{n}\right|+n\left|b_{n}\right|\right) \leq 1
$$

Moreover,

$$
\begin{aligned}
\left|h^{\prime}(z)\right|-\left|g^{\prime}(z)\right| & \geq 1-\sum_{n=2}^{\infty} n\left|a_{n}\right||z|^{n}-\sum_{n=2}^{\infty} n\left|b_{n}\right||z|^{n} \geq 1-|z| \sum_{n=2}^{\infty}\left(n\left|a_{n}\right|+n\left|b_{n}\right|\right) \\
& \geq 1-\frac{|z|}{B-A} \sum_{n=2}^{\infty}\left(\left|\gamma_{n} a_{n}\right|+\left|\delta_{n} b_{n}\right|\right) \geq 1-|z|>0 \quad(z \in \mathbb{U}) .
\end{aligned}
$$

Thus, by (13) the function $f$ is locally univalent and sense preserving in $\mathbb{U}$. Also, for $z_{1}, z_{2} \in \mathbb{U}, z_{1} \neq z_{2}$, we have

$$
\left|\frac{z_{1}^{n}-z_{2}^{n}}{z_{1}-z_{2}}\right|=\left|\sum_{k=1}^{n} z_{1}^{k-1} z_{2}^{n-k}\right| \leq \sum_{k=1}^{n}\left|z_{1}\right|^{k-1}\left|z_{2}\right|^{n-k}<n \quad(n=2,3, \ldots) .
$$

Hence, by (15) we get

$$
\begin{aligned}
\left|f\left(z_{1}\right)-f\left(z_{2}\right)\right| & \geq\left|h\left(z_{1}\right)-h\left(z_{2}\right)\right|-\left|g\left(z_{1}\right)-g\left(z_{2}\right)\right| \\
& \geq\left|z_{1}-z_{2}-\sum_{n=2}^{\infty} a_{n}\left(z_{1}^{n}-z_{2}^{n}\right)\right|-\left|\sum_{n=2}^{\infty} \overline{b_{n}} \overline{\left(z_{1}^{n}-z_{2}^{n}\right)}\right| \\
& \geq\left|z_{1}-z_{2}\right|-\sum_{n=2}^{\infty}\left|a_{n}\right|\left|z_{1}^{n}-z_{2}^{n}\right|-\sum_{n=2}^{\infty}\left|b_{n}\right|\left|z_{1}^{n}-z_{2}^{n}\right| \\
& =\left|z_{1}-z_{2}\right|\left(1-\sum_{n=2}^{\infty}\left(\left|a_{n}\right|-\left|b_{n}\right|\right)\left|\frac{z_{1}^{n}-z_{2}^{n}}{z_{1}-z_{2}}\right|\right)>0,
\end{aligned}
$$

which gives $f\left(z_{1}\right) \neq f\left(z_{2}\right)$ and, in consequence, $f \in \mathcal{S}_{\mathcal{H}}$. Thus, we need only prove that the function $f$ satisfies (4), i.e., there exists a complex-valued function $\omega, \omega(0)=0,|\omega(z)|<1(z \in \mathbb{U})$ such that

$$
\frac{D_{\mathcal{H}}(f * \varphi)(z)}{(f * \varphi)(z)}=\frac{1+A \omega(z)}{1+B \omega(z)} \quad(z \in \mathbb{U})
$$

or equivalently

$$
\left|\frac{D_{\mathcal{H}}(f * \varphi)(z)-(f * \varphi)(z)}{B D_{\mathcal{H}}(f * \varphi)(z)-A(f * \varphi)(z)}\right|<1 \quad(z \in \mathbb{U}) .
$$

Therefore, it is suffice to prove that 


$$
\left|D_{\mathcal{H}}(f * \varphi)(z)-(f * \varphi)(z)\right|-\left|B D_{\mathcal{H}}(f * \varphi)(z)-A(f * \varphi)(z)\right|<0 \quad(z \in \mathbb{U} \backslash\{0\}) .
$$

Indeed, letting $|z|=r(0<r<1)$ we have

$$
\begin{aligned}
\mid D_{\mathcal{H}} & (f * \varphi)(z)-(f * \varphi)(z)|-| B D_{\mathcal{H}}(f * \varphi)(z)-A(f * \varphi)(z) \mid \\
= & \left|\sum_{n=2}^{\infty}(n-1) u_{n} a_{n} z^{n}-\sum_{n=2}^{\infty}(n+1) \bar{v}_{n} \bar{b}_{n} \bar{z}^{n}\right| \\
& -\left|(B-A) z+\sum_{n=2}^{\infty}(B n-A) u_{n} a_{n} z^{n}-\sum_{n=2}^{\infty}(B n+A) \bar{v}_{n} \bar{b}_{n} \bar{z}^{n}\right| \\
\leq & \sum_{n=2}^{\infty}(n-1)\left|u_{n} a_{n}\right| r^{n}+\sum_{n=2}^{\infty}(n+1)\left|v_{n} b_{n}\right| r^{n}-(B-A) r \\
& +\sum_{n=2}^{\infty}(B n-A)\left|u_{n} a_{n}\right| r^{n}+\sum_{n=2}^{\infty}(B n+A)\left|v_{n} b_{n}\right| r^{n} \\
\leq & r\left\{\sum_{n=2}^{\infty}\left(\left|\gamma_{n}\right|\left|a_{n}\right|+\left|\delta_{n}\right|\left|b_{n}\right|\right) r^{n-1}-(B-A)\right\}<0 .
\end{aligned}
$$

whence $f \in \mathcal{W}_{\mathcal{H}}(\varphi ; A, B)$.

Now, we show that the condition (11) is also a sufficient condition for a function $f \in \mathcal{T}^{\eta}(\varphi)$ to be in the class $\mathcal{W}_{\mathcal{H}}^{\eta}(\varphi ; A, B)$.

Theorem 4 Let $f \in \mathcal{H}_{0}$ be a function of the form (1). Then $f \in \mathcal{W}_{\mathcal{H}}^{\eta}(\varphi ; A, B)$ if and only if the condition (11) holds true.

Proof In view of Theorem 3, we have to prove "only if" part, i.e., each function from the class $\mathcal{W}_{\mathcal{H}}^{\eta}(\varphi ; A, B)$ satisfies the condition (11). If $f \in \mathcal{W}_{\mathcal{H}}^{\eta}(\varphi ; A, B)$, then it is of the form (1) with (7) and satisfies (16) or equivalently

$$
\left|\frac{\sum_{n=2}^{\infty}\left\{(n-1) \eta^{1-n}\left|u_{n}\right|\left|a_{n}\right| z^{n}+(n+1) \eta^{1+n}\left|v_{n}\right|\left|b_{n}\right| \bar{z}^{n}\right\}}{(B-A) z-\sum_{n=2}^{\infty}\left\{(B n-A) \eta^{1-n}\left|u_{n}\right|\left|a_{n}\right| z^{n}+(B n+A) \eta^{1-n}\left|v_{n}\right|\left|b_{n}\right| \bar{z}^{n}\right\}}\right|<1 \quad(z \in \mathbb{U}) .
$$

Thus, letting $z=r \eta(0 \leq r<1)$, we have

$$
\frac{\sum_{n=2}^{\infty}\left\{(n-1)\left|u_{n}\right|\left|a_{n}\right|+(n+1)\left|v_{n}\right|\left|b_{n}\right| r^{n-1}\right\}}{(B-A)-\sum_{n=2}^{\infty}\left\{(B n-A)\left|u_{n}\right|\left|a_{n}\right|+(B n+A)\left|v_{n}\right|\left|b_{n}\right| r^{n-1}\right\}}<1 .
$$


It is clear that the denominator of the left-hand side cannot vanish for $r \in\langle 0,1)$. Moreover, it is positive for $r=0$, and in consequence for $r \in\langle 0,1)$. Thus, by (17) we have

$$
\sum_{n=2}^{\infty}\left(\left|\gamma_{n} a_{n}\right|+\left|\delta_{n} b_{n}\right|\right) r^{n-1}<B-A \quad(0 \leq r<1) .
$$

The sequence of partial sums $\left\{S_{n}\right\}$ associated with the series $\sum_{n=2}^{\infty}\left(\left|\gamma_{n} a_{n}\right|+\left|\delta_{n} b_{n}\right|\right)$ is a nondecreasing sequence. Moreover, by (18) it is bounded by $B-A$. Hence, the sequence $\left\{S_{n}\right\}$ is convergent and

$$
\sum_{n=2}^{\infty}\left(\left|\gamma_{n} a_{n}\right|+\left|\delta_{n} b_{n}\right|\right)=\lim _{n \rightarrow \infty} S_{n} \leq B-A,
$$

which yields the assertion (11).

The following result may be proved in the same way as Theorem 4 .

Theorem 5 Let $f \in \mathcal{H}_{0}$ be a function of the form (1). Then $f \in \mathcal{V}_{\mathcal{H}}^{\eta}(\varphi ; A, B)$ if and only if

$$
\sum_{n=2}^{\infty}\left(\left|u_{n}\right|\left|a_{n}\right|+\left|v_{n}\right|\left|b_{n}\right|\right) \leq \frac{B-A}{1+B} .
$$

By Theorems 4 and 5 we have the following corollary.

Corollary 1 Let $a=\frac{1+A}{1+B}$ and

$$
\begin{aligned}
& \phi(z)=z+\sum_{n=k}^{\infty}\left(\frac{1}{n-a} z^{n}+\frac{1}{n+a} \bar{z}^{n}\right) \quad(z \in \mathbb{U}), \\
& \omega(z)=z+\sum_{n=k}^{\infty}\left((n-a) z^{n}+(n+a) \bar{z}^{n}\right) \quad(z \in \mathbb{U}) .
\end{aligned}
$$

Then

$$
\mathcal{V}_{\mathcal{H}}^{\eta}(\varphi ; A, B)=\mathcal{W}_{\mathcal{T}}^{\eta}(\varphi * \phi ; A, B), \mathcal{W}_{\mathcal{H}}^{\eta}(\varphi ; A, B)=\mathcal{V}_{\mathcal{T}}^{\eta}(\varphi * \omega ; A, B)
$$

In particular,

$$
\mathcal{W}_{\mathcal{T}}^{\eta}(\varphi ;-1, B)=\mathcal{V}_{\mathcal{T}}^{\eta}\left(D_{\mathcal{H}}(\varphi) ;-1, B\right)
$$

\section{Extreme points}

Let us consider the usual topology on $\mathcal{H}$ defined by a metric in which a sequence $\left\{f_{n}\right\}$ in $\mathcal{H}$ converges to $f$ if and only if it converges to $f$ uniformly on each compact subset of $\mathbb{U}$. It follows from the theorems of Weierstrass and Montel that this topological space is complete. 
Let $\mathcal{F}$ be a subclass of the class $\mathcal{H}$. A functions $f \in \mathcal{F}$ is called an extreme point of $\mathcal{F}$ if the condition

$$
f=\gamma f_{1}+(1-\gamma) f_{2} \quad\left(f_{1}, f_{2} \in \mathcal{F}, 0<\gamma<1\right)
$$

implies $f_{1}=f_{2}=f$. We shall use the notation $E \mathcal{F}$ to denote the set of all extreme points of $\mathcal{F}$. It is clear that $E \mathcal{F} \subset \mathcal{F}$.

We say that $\mathcal{F}$ is locally uniformly bounded if for each $r, 0<r<1$, there is a real constant $M=M(r)$ so that

$$
|f(z)| \leq M \quad(f \in \mathcal{F},|z| \leq r) .
$$

We say that a class $\mathcal{F}$ is convex if

$$
\gamma f+(1-\gamma) g \in \mathcal{F} \quad(f, g \in \mathcal{F}, 0 \leq \gamma \leq 1)
$$

Moreover, we define the closed convex hull of $\mathcal{F}$ as the intersection of all closed convex subsets of $\mathcal{H}$ that contain $\mathcal{F}$. We denote the closed convex hull of $\mathcal{F}$ by $\overline{c o} \mathcal{F}$.

A real-valued functional $\mathcal{J}: \mathcal{H} \rightarrow \mathbb{R}$ is called convex on a convex class $\mathcal{F} \subset \mathcal{H}$ if

$$
\mathcal{J}(\gamma f+(1-\gamma) g) \leq \gamma \mathcal{J}(f)+(1-\gamma) \mathcal{J}(g) \quad(f, g \in \mathcal{F}, 0 \leq \gamma \leq 1) .
$$

The Krein-Milman theorem (see [24]) is fundamental in the theory of extreme points. In particular, it implies the following lemma.

Lemma 1 [12, pp. 45] Let $\mathcal{F}$ be a non-empty compact convex subclass of the class $\mathcal{H}$ and $\mathcal{J}: \mathcal{H} \rightarrow \mathbb{R}$ be a real-valued, continuous and convex functional on $\mathcal{F}$. Then

$$
\max \{\mathcal{J}(f): f \in \mathcal{F}\}=\max \{\mathcal{J}(f): f \in E \mathcal{F}\}
$$

Since $\mathcal{H}$ is a complete metric space, Montel's theorem (see [26]) implies the following lemma.

Lemma 2 A class $\mathcal{F} \subset \mathcal{H}$ is compact if and only if $\mathcal{F}$ is closed and locally uniformly bounded.

Theorem 6 The class $\mathcal{W}_{\mathcal{H}}^{\eta}(\varphi ; A, B)$ is convex and compact subset of $\mathcal{H}$.

Proof Let $f_{k} \in \mathcal{H}, k \in \mathbb{N}$, be functions of the form

$$
f_{k}(z)=\sum_{n=0}^{\infty} a_{k, n} z^{n}+\sum_{n=1}^{\infty} \overline{b_{k, n} z^{n}} \quad(z \in \mathbb{U})
$$

Let $f_{1}, f_{2} \in \mathcal{W}_{\mathcal{T}}^{\eta}(\varphi ; A, B)$ be functions of the form (19), $0 \leq \gamma \leq 1$. Since $\gamma f_{1}(z)+(1-\gamma) f_{2}(z)=z+\sum_{n=2}^{\infty}\left\{\left(\gamma a_{1, n}+(1-\gamma) a_{2, n}\right) z^{n}+\overline{\left(\gamma b_{1, n}+(1-\gamma) b_{2, n}\right) z^{n}}\right\}$, 
and by Theorem 4 we have

$$
\begin{aligned}
& \sum_{n=2}^{\infty}\left\{\left|\gamma_{n}\right|\left|\gamma a_{1, n}+(1-\gamma) a_{2, n}\right|+\left|\delta_{n}\right|\left|\gamma b_{1, n}+(1-\gamma) b_{2, n} z^{n}\right|\right\} \\
& \leq \gamma \sum_{n=2}^{\infty}\left\{\left|\gamma_{n} a_{1, n}\right|+\left|\delta_{n} b_{1, n}\right|\right\}+(1-\gamma) \sum_{n=2}^{\infty}\left\{\left|\gamma_{n} a_{2, n}\right|+\left|\delta_{n} b_{2, n}\right|\right\} \\
& \leq \gamma(B-A)+(1-\gamma)(B-A)=B-A,
\end{aligned}
$$

the function $\phi=\gamma f_{1}+(1-\gamma) f_{2}$ belongs to the class $\mathcal{W}_{\mathcal{T}}^{\eta}(\varphi ; A, B)$. Hence, the class is convex. Furthermore, for $f \in \mathcal{W}_{\mathcal{T}}^{\eta}(\varphi ; A, B),|z| \leq r, 0<r<1$, we have

$$
|f(z)| \leq r+\sum_{n=2}^{\infty}\left(\left|a_{n}\right|+\left|b_{n}\right|\right) r^{n} \leq r+\sum_{n=2}^{\infty}\left(\gamma_{n}\left|a_{n}\right|+\delta_{n}\left|b_{n}\right|\right) \leq r+(B-A)
$$

Thus, we conclude that the class $\mathcal{W}_{\mathcal{T}}^{\eta}(\varphi ; A, B)$ is locally uniformly bounded. By Lemma 2, we only need to show that it is closed, i.e., if $f_{k} \in \mathcal{W}_{\mathcal{T}}^{\eta}(\varphi ; A, B) \quad(k \in \mathbb{N})$ and $f_{k} \rightarrow f$, then $f \in \mathcal{W}_{\mathcal{T}}^{\eta}(\varphi ; A, B)$. Let $f_{k}$ and $f$ be given by (19) and (1), respectively. Using Theorem 4 we have

$$
\sum_{n=2}^{\infty}\left(\left|\gamma_{n} a_{k, n}\right|+\left|\delta_{n} b_{k, n}\right|\right) \leq B-A \quad(k \in \mathbb{N})
$$

Since $f_{k} \rightarrow f$, we conclude that $\left|a_{k, n}\right| \rightarrow\left|a_{n}\right|$ and $\left|b_{k, n}\right| \rightarrow\left|b_{n}\right|$ as $k \rightarrow \infty(n \in \mathbb{N})$. The sequence of partial sums $\left\{S_{n}\right\}$ associated with the series $\sum_{n=2}^{\infty}\left(\left|\gamma_{n} a_{n}\right|+\left|\delta_{n} b_{n}\right|\right)$ is a nondecreasing sequence. Moreover, by (21) it is bounded by $B-A$. Therefore, the sequence $\left\{S_{n}\right\}$ is convergent and

$$
\sum_{n=2}^{\infty}\left(\left|\gamma_{n} a_{n}\right|+\left|\delta_{n} b_{n}\right|\right)=\lim _{n \rightarrow \infty} S_{n} \leq B-A
$$

This gives the condition (11), and, in consequence, $f \in \mathcal{W}_{\mathcal{T}}^{\eta}(\varphi ; A, B)$, which completes the proof.

\section{Theorem 7}

$$
\operatorname{E\mathcal {W}}_{\mathcal{H}}^{\eta}(\varphi ; A, B)=\left\{h_{n}: n \in \mathbb{N}\right\} \cup\left\{g_{n}: n \in\{2,3 \ldots\}\right\},
$$

where $h_{1}(z)=z$ and

$$
h_{n}(z)=z-\frac{B-A}{\eta^{n-1} \gamma_{n}} z^{n}, \quad g_{n}(z)=z+\frac{B-A}{\eta^{-n-1} \delta_{n}} \bar{z}^{n} \quad(z \in \mathbb{U}) .
$$


Proof Suppose that $0<\gamma<1$ and

$$
g_{n}=\gamma f_{1}+(1-\gamma) f_{2}
$$

where $f_{1}, f_{2} \in \mathcal{W}_{\mathcal{T}}^{\eta}(\varphi ; A, B)$ are functions of the form (19). Then, by (11) we have $\left|b_{1, n}\right|=\left|b_{2, n}\right|=\frac{B-A}{\left|\delta_{n}\right|}$, and, in consequence, $a_{1, k}=a_{2, k}=0$ for $k \in\{2,3 \ldots\}$ and $b_{1, k}=b_{2, k}=0$ for $k \in\{2,3 \ldots\} \backslash\{n\}$. It follows that $g_{n}=f_{1}=f_{2}$, and consequently $g_{n} \in E \mathcal{W}_{\mathcal{T}}^{\eta}(\varphi ; A, B)$. Similarly, we verify that the functions $h_{n}$ of the form (22) are the extreme points of the class $\mathcal{W}_{\mathcal{H}}^{\eta}(\varphi ; A, B)$. Now, suppose that a function $f$ belongs to the set $\operatorname{EW}_{\mathcal{H}}^{\eta}(\varphi ; A, B)$ and $f$ is not of the form (22). Then there exists $m \in\{2,3, \ldots\}$ such that

$$
0<\left|a_{m}\right|<\frac{B-A}{\left|\gamma_{m}\right|} \quad \text { or } \quad 0<\left|b_{m}\right|<\frac{B-A}{\left|\delta_{m}\right|} .
$$

If $0<\left|a_{m}\right|<\frac{B-A}{\left|\gamma_{m}\right|}$, then putting

$$
\gamma=\frac{\left|\gamma_{m} a_{m}\right|}{B-A}, \quad \varphi=\frac{1}{1-\gamma}\left(f-\gamma h_{m}\right),
$$

we have that $0<\gamma<1, h_{m}, \varphi \in \mathcal{W}_{\mathcal{H}}^{\eta}(\varphi ; A, B), h_{m} \neq \varphi$ and

$$
f=\gamma h_{m}+(1-\gamma) \varphi
$$

Thus, $f \notin E \mathcal{W}_{\mathcal{H}}^{\eta}(\varphi ; A, B)$. Similarly, if $0<\left|b_{m}\right|<\frac{B-A}{\left|\delta_{n}\right|}$, then putting

$$
\gamma=\frac{\left|\delta_{m} b_{m}\right|}{B-A}, \quad \phi=\frac{1}{1-\gamma}\left(f-\gamma g_{m}\right)
$$

we have that $0<\gamma<1, g_{m}, \phi \in \mathcal{W}_{\mathcal{H}}^{\eta}(\varphi ; A, B), g_{m} \neq \phi$ and

$$
f=\gamma g_{m}+(1-\gamma) \phi \text {. }
$$

It follows that $f \notin E \mathcal{W}_{\mathcal{H}}^{\eta}(\varphi ; A, B)$, and the proof is completed.

\section{Radii of starlikeness and convexity}

We say that a function $f \in \mathcal{H}_{0}$ is starlike of order $\alpha$ in $\mathbb{U}(r)$ if

$$
\frac{\partial}{\partial t}\left(\arg f\left(\rho e^{i t}\right)\right)>\alpha, \quad 0 \leq t \leq 2 \pi, \quad 0<\rho<r<1 .
$$


Analogously, we say that a function $f \in \mathcal{H}_{0}$ is convex of order $\alpha$ in $\mathbb{U}(r)$ if

$$
\frac{\partial}{\partial t}\left(\arg \frac{\partial}{\partial t} f\left(\rho e^{i t}\right)\right)>\alpha, \quad 0 \leq t \leq 2 \pi, 0<\rho<r<1 .
$$

It is easy to verify that for a function $f \in \mathcal{T}(\varphi)$ the condition (23) is equivalent to the following

$$
\operatorname{Re} \frac{D_{\mathcal{H}} f(z)}{f(z)}>\alpha \quad(z \in \mathbb{U}(r))
$$

or equivalently

$$
\left|\frac{D_{\mathcal{H}} f(z)-(1+\alpha) f(z)}{D_{\mathcal{H}} f(z)+(1-\alpha) f(z)}\right|<1 \quad(z \in \mathbb{U}(r))
$$

Let $\mathcal{B}$ be a subclass of the class $\mathcal{H}_{0}$. We define the radius of starlikeness $R_{\alpha}^{*}(\mathcal{B})$ and the radius of convexity $R_{\alpha}^{c}(\mathcal{B})$ for the class $\mathcal{B}$ by

$$
\begin{aligned}
& R_{\alpha}^{*}(\mathcal{B}):=\inf _{f \in \mathcal{B}}(\sup \{r \in(0,1]: f \text { is starlike of order in } \mathbb{U}(r)\}), \\
& R_{\alpha}^{c}(\mathcal{B}):=\inf _{f \in \mathcal{B}}(\sup \{r \in(0,1]: f \text { is convex of order in } \mathbb{U}(r)\}) .
\end{aligned}
$$

Theorem 8 The radius of starlikeness of order $\alpha$ for the class $\mathcal{W}_{\mathcal{H}}^{\eta}(\varphi ; A, B)$ is given by

$$
R_{\alpha}^{*}\left(\mathcal{W}_{\mathcal{H}}^{\eta}(\varphi ; A, B)\right)=\inf _{n \geq 2}\left(\frac{1-\alpha}{B-A} \min \left\{\frac{\left|\gamma_{n}\right|}{n-\alpha}, \frac{\left|\delta_{n}\right|}{n+\alpha}\right\}\right)^{\frac{1}{n-1}}
$$

where $\gamma_{n}$ and $\delta_{n}$ are defined by (12).

Proof Let $f \in \mathcal{W}_{\mathcal{H}}^{\eta}(\varphi ; A, B)$ be of the form (1) with (7). Then, for $|z|=r<1$ we have

$$
\begin{aligned}
\left|\frac{D_{\mathcal{H}} f(z)-(1+\alpha) f(z)}{D_{\mathcal{H}} f(z)+(1-\alpha) f(z)}\right| & =\left|\frac{-\alpha z+\sum_{n=2}^{\infty}\left((n-1-\alpha)\left|a_{n}\right| z^{n}-(n+1+\alpha)\left|b_{n}\right| \bar{z}^{n}\right)}{(2-\alpha) z+\sum_{n=2}^{\infty}\left((n+1-\alpha)\left|a_{n}\right| z^{n}-(n-1+\alpha)\left|b_{n}\right| \bar{z}^{n}\right)}\right| \\
& \leq \frac{\alpha+\sum_{n=2}^{\infty}\left((n-1-\alpha)\left|a_{n}\right|+(n+1+\alpha)\left|b_{n}\right|\right) r^{n-1}}{2-\alpha-\sum_{n=2}^{\infty}\left((n+1-\alpha)\left|a_{n}\right|+(n-1+\alpha)\left|b_{n}\right|\right) r^{n-1}}
\end{aligned}
$$

Thus, the condition (24) is true if and only if

$$
\sum_{n=2}^{\infty}\left(\frac{n-\alpha}{1-\alpha}\left|a_{n}\right|+\frac{n+\alpha}{1-\alpha}\left|b_{n}\right|\right) r^{n-1} \leq 1
$$


By Theorem 1, we have

$$
\sum_{n=2}^{\infty}\left(\frac{\left|\gamma_{n}\right|}{B-A}\left|a_{n}\right|+\frac{\left|\delta_{n}\right|}{B-A}\left|b_{n}\right|\right) \leq 1
$$

where $\gamma_{n}$ and $\delta_{n}$ are defined by (12). Thus, the condition (26) is true if

$$
\frac{n-\alpha}{1-\alpha} r^{n-1} \leq \frac{\left|\gamma_{n}\right|}{B-A}, \frac{n+\alpha}{1-\alpha} r^{n-1} \leq \frac{\left|\delta_{n}\right|}{B-A} \quad(n=2,3 \ldots),
$$

that is, if

$$
r \leq\left(\frac{1-\alpha}{B-A} \min \left\{\frac{\left|\gamma_{n}\right|}{n-\alpha}, \frac{\left|\delta_{n}\right|}{n+\alpha}\right\}\right)^{\frac{1}{n-1}} \quad(n=2,3 \ldots) .
$$

It follows that the function $f$ is starlike of order $\alpha$ in the disk $U\left(r^{*}\right)$, where $r^{*}$

$$
r^{*}:=\inf _{n \geq 2}\left(\frac{1-\alpha}{B-A} \min \left\{\frac{\left|\gamma_{n}\right|}{n-\alpha}, \frac{\left|\delta_{n}\right|}{n+\alpha}\right\}\right)^{\frac{1}{n-1}} .
$$

The functions $h_{n}, g_{n}$ of the form (22) realize equality in (27), and the radius $r^{*}$ cannot be larger. Thus we have (25).

The following result may be proved in much the same way as Theorem 8 .

Theorem 9 The radius of convexity of order $\alpha$ for the class $\mathcal{W}_{\mathcal{H}}^{\eta}(\varphi ; A, B)$ is given by

$$
R_{\alpha}^{c}\left(\mathcal{W}_{\mathcal{H}}^{\eta}(\varphi ; A, B)\right)=\inf _{n \geq 2}\left(\frac{1-\alpha}{B-A} \min \left\{\frac{\left|\gamma_{n}\right|}{n(n-\alpha)}, \frac{\left|\delta_{n}\right|}{n(n+\alpha)}\right\}\right)^{\frac{1}{n-1}}
$$

where $\gamma_{n}$ and $\delta_{n}$ are defined by (12).

\section{Applications}

It is clear that if the class $\mathcal{F}=\left\{f_{n} \in \mathcal{H}: n \in \mathbb{N}\right\}$ is locally uniformly bounded, then

$$
\overline{c o} \mathcal{F}=\left\{\sum_{n=1}^{\infty} \gamma_{n} f_{n}: \quad \sum_{n=1}^{\infty} \gamma_{n}=1, \gamma_{n} \geq 0(n \in \mathbb{N})\right\} .
$$

Thus, by Theorem 8 we have the following corollary.

\section{Corollary 2}

$\mathcal{W}_{\mathcal{H}}^{\eta}(\varphi ; A, B)=\left\{\sum_{n=1}^{\infty}\left(\gamma_{n} h_{n}+\delta_{n} g_{n}\right): \sum_{n=1}^{\infty}\left(\gamma_{n}+\delta_{n}\right)=1, \delta_{1}=0, \gamma_{n}, \delta_{n} \geq 0 \quad(n \in \mathbb{N})\right\}$,

where $h_{n}, g_{n}$ are defined by (22). 
For each fixed value of $n \in \mathbb{N}, z \in \mathbb{U}$, the following real-valued functionals are continuous and convex on $\mathcal{H}$ :

$$
\mathcal{J}(f)=\left|a_{n}\right|, \_\mathcal{J}(f)=\left|b_{n}\right|, \mathcal{J}(f)=|f(z)| \mathcal{J}(f)=\left|D_{\mathcal{H}} f(z)\right| \quad(f \in \mathcal{H})
$$

Moreover, for $\gamma \geq 1,0<r<1$, the real-valued functional

$$
\mathcal{J}(f)=\left(\frac{1}{2 \pi} \int_{0}^{2 \pi}\left|f\left(r e^{i \theta}\right)\right|^{\gamma} \mathrm{d} \theta\right)^{1 / \gamma} \quad(f \in \mathcal{H})
$$

is also continuous and convex on $\mathcal{H}$.

Therefore, by Lemma 1 and Theorem 8 we have the following corollaries.

Corollary 3 Let $f \in \mathcal{W}_{\mathcal{H}}^{\eta}(\varphi ; A, B)$ be a function of the form (1). Then

$$
\left|a_{n}\right| \leq \frac{B-A}{\left|\gamma_{n}\right|},\left|b_{n}\right| \leq \frac{B-A}{\left|\delta_{n}\right|} \quad(n=2,3, \ldots),
$$

where $\gamma_{n}, \delta_{n}$ are defined by (12). The result is sharp. The functions $h_{n}, g_{n}$ of the form (22) are the extremal functions.

Corollary 4 Let $f \in \mathcal{W}_{\mathcal{H}}^{\eta}(\varphi ; A, B),|z|=r<1$. Then

$$
\begin{aligned}
& r-\frac{B-A}{\left|u_{2}\right|(1+2 B-A)} r^{2} \leq|f(z)| \leq r+\frac{B-A}{\left|u_{2}\right|(1+2 B-A)} r^{2}, \\
& r-\frac{2(B-A)}{\left|u_{2}\right|(1+2 B-A)} r^{2} \leq\left|D_{\mathcal{H}} f(z)\right| \leq r+\frac{2(B-A)}{\left|u_{2}\right|(1+2 B-A)} r^{2}
\end{aligned}
$$

where $u_{2}$ is defined by (2). The result is sharp. The function $h_{2}$ of the form (22) is the extremal function.

Corollary 5 Let $0<r<1, \gamma \geq 1$. If $f \in \mathcal{W}_{\mathcal{H}}^{\eta}(\varphi ; A, B)$, then

$$
\begin{aligned}
& \frac{1}{2 \pi} \int_{0}^{2 \pi}\left|f\left(r e^{i \theta}\right)\right|^{\gamma} \mathrm{d} \theta \leq \frac{1}{2 \pi} \int_{0}^{2 \pi}\left|h_{2}\left(r e^{i \theta}\right)\right|^{\lambda} \mathrm{d} \theta, \\
& \frac{1}{2 \pi} \int_{0}^{2 \pi}\left|D_{\mathcal{H}} f(z)\right|^{\gamma} \mathrm{d} \theta \leq \frac{1}{2 \pi} \int_{0}^{2 \pi}\left|D_{\mathcal{H}} h_{2}\left(r e^{i \theta}\right)\right|^{\gamma} \mathrm{d} \theta,
\end{aligned}
$$

where $h_{2}$ is the function defined by (22).

The following covering result follows from Corollary 4 . 
Corollary 6 If $f \in \mathcal{W}_{\mathcal{H}}^{\eta}(\varphi ; A, B)$, then $\mathbb{U}(r) \subset f(\mathbb{U})$, where

$$
r=1-\frac{B-A}{\left|u_{2}\right|(1+2 B-A)} .
$$

Using Corollary 1 and the results above we obtain corollaries listed below.

\section{Corollary 7}

$$
\begin{aligned}
& R_{\alpha}^{*}\left(\mathcal{V}_{\mathcal{H}}^{\eta}(\varphi ; A, B)\right)=\inf _{n \geq 2}\left(\frac{1+B}{B-A} \min \left\{\frac{1-\alpha}{n-\alpha} n\left|u_{n}\right|, \frac{1-\alpha}{n+\alpha} n\left|v_{n}\right|\right\}\right)^{\frac{1}{n-1}} \\
& R_{\alpha}^{c}\left(\mathcal{V}_{\mathcal{H}}^{\eta}(\varphi ; A, B)\right)=\inf _{n \geq 2}\left(\frac{1+B}{B-A} \min \left\{\frac{1-\alpha}{n-\alpha}\left|u_{n}\right|, \frac{1-\alpha}{n+\alpha}\left|v_{n}\right|\right\}\right)^{\frac{1}{n-1}},
\end{aligned}
$$

where $u_{n}$ and $v_{n}$ are defined by (2).

Corollary 8 The class $\mathcal{V}_{\mathcal{H}}^{\eta}(\varphi ; A, B)$ is convex and compact subset of $\mathcal{H}$. Moreover,

$$
E \mathcal{V}_{\mathcal{H}}^{\eta}(\varphi ; A, B)=\left\{h_{n}: n \in \mathbb{N}\right\} \cup\left\{g_{n}: n \in\{2,3 \ldots\}\right\}
$$

and

$$
\mathcal{V}_{\mathcal{H}}^{\eta}(\varphi ; A, B)=\left\{\sum_{n=1}^{\infty}\left(\gamma_{n} h_{n}+\delta_{n} g_{n}\right): \sum_{n=1}^{\infty}\left(\gamma_{n}+\delta_{n}\right)=1, \delta_{1}=0, \gamma_{n}, \delta_{n} \geq 0 \quad(n \in \mathbb{N})\right\}
$$

where $h_{1}(z)=z$ and

$$
h_{n}(z)=z-\frac{(B-A) \eta^{1-n}}{(1+B) u_{n}} z^{n}, g_{n}(z)=z+\frac{(B-A) \eta^{1+n}}{(1+B) v_{n}} \bar{z}^{n} \quad(z \in \mathbb{U}) .
$$

Corollary 9 Let $f \in \mathcal{V}_{\mathcal{H}}^{\eta}(\varphi ; A, B)$ be a function of the form (1). Then us assume (3). If $f \in \mathcal{V}_{\mathcal{H}}^{\eta}(\varphi ; A, B)$, then $\mathbb{U}(r) \subset f(\mathbb{U})$, where

$$
\begin{aligned}
& \left|a_{n}\right| \leq \frac{B-A}{(1+B)\left|u_{n}\right|}, \quad\left|b_{n}\right| \leq \frac{B-A}{(1+B)\left|v_{n}\right|} \quad(n=2,3, \ldots), \\
& r-\frac{B-A}{(1+B)\left|u_{2}\right|} r^{2} \leq|f(z)| \leq r+\frac{B-A}{(1+B)\left|u_{2}\right|} r^{2} \quad(|z|=r<1), \\
& r-\frac{2(B-A)}{(1+B)\left|u_{2}\right|} r^{2} \leq\left|D_{\mathcal{H}} f(z)\right| \leq r+\frac{2(B-A)}{(1+B)\left|u_{2}\right|} r^{2} \quad(|z|=r<1), \\
& \quad \frac{1}{2 \pi} \int_{0}^{2 \pi}\left|f\left(r e^{i \theta}\right)\right|^{\gamma} \mathrm{d} \theta \leq \frac{1}{2 \pi} \int_{0}^{2 \pi}\left|h_{2}\left(r e^{i \theta}\right)\right|^{\lambda} \mathrm{d} \theta,
\end{aligned}
$$




$$
\frac{1}{2 \pi} \int_{0}^{2 \pi}\left|D_{\mathcal{H}} f\left(r e^{i \theta}\right)\right|^{\gamma} \mathrm{d} \theta \leq \frac{1}{2 \pi} \int_{0}^{2 \pi}\left|D_{\mathcal{H}} h_{2}\left(r e^{i \theta}\right)\right|^{\gamma} \mathrm{d} \theta
$$

where $u_{n}$ and $v_{n}$ are defined by (2). The results are sharp. The functions $h_{n}, g_{n}$ of the form (32) are the extremal functions.

Corollary 10 Let

$$
r=1-\frac{B-A}{(1+B)\left|u_{2}\right|}
$$

\section{Concluding remarks}

We conclude the paper by observing that, in view of the subordination relation (5) and (6), choosing the function $\varphi$ and the parameters $A, B$ we can consider new and also well-known classes of functions. In particular, the classes

$$
\mathcal{W}_{\mathcal{H}}^{\eta}(\varphi ; \alpha):=\mathcal{W}_{\mathcal{H}}^{\eta}(\varphi ; 2 \alpha-1, B), \quad \mathcal{W}_{\mathcal{H}}^{\eta}(\varphi ; \alpha):=\mathcal{W}_{\mathcal{H}}^{\eta}(\varphi ; 2 \alpha-1, B)
$$

contain functions $f \in \mathcal{S}_{H}$ which satisfy the conditions

$$
\begin{aligned}
& \operatorname{Re} \frac{D_{\mathcal{H}}(\varphi * f)(z)}{(\varphi * f)(z)}>\alpha \quad(z \in \mathbb{U}), \\
& \operatorname{Re} \frac{(\varphi * f)(z)}{z}>\alpha \quad(z \in \mathbb{U}),
\end{aligned}
$$

respectively.

The considered classes are defined using the convolution $\varphi * f$ or equivalently by the linear operator

$$
J_{\varphi}: \mathcal{H} \rightarrow \mathcal{H}, \quad J_{\varphi}(f)=\varphi * f
$$

By choosing the function $\varphi$, we can obtain a lot of important linear operators, and in consequence new and also well-known classes of functions.

Let $q, s \in \mathbb{N}_{0}, q \leq s+1$. For complex parameters $\alpha_{1}, \ldots, \alpha_{q}$ and $\beta_{1}, \ldots, \beta_{s}$, where $\beta_{j} \neq 0,-1,-2, \ldots$, we define the generalized hypergeometric function by

$$
F_{q, s}(z)={ }_{q} F_{s}\left(\alpha_{1}, \ldots, \alpha_{q} ; \beta_{1}, \ldots, \beta_{s} ; z\right):=\sum_{n=0}^{\infty} \frac{\left(\alpha_{1}\right)_{n} \cdots\left(\alpha_{q}\right)_{n}}{\left(\beta_{1}\right)_{n} \cdots\left(\beta_{s}\right)_{n}} \frac{z^{n}}{n !} \quad(z \in \mathbb{U}),
$$

where $(\lambda)_{n}$ is the Pochhammer symbol defined, in terms of the Gamma function $\Gamma$, by

$$
(\lambda)_{n}:=\frac{\Gamma(\lambda+n)}{\Gamma(\lambda)}= \begin{cases}1 & (n=0) \\ \lambda(\lambda+1) \cdots(\lambda+n-1) & (n \in \mathbb{N}) .\end{cases}
$$

Corresponding to the generalized hypergeometric function, Dziok and Srivastava [16] introduced a linear operator on the class of analytic functions, defined by the convolution: 


$$
H_{q, s} f(z):=\left(z F_{q, s}(z)\right) * f(z) \quad(z \in \mathbb{U}) .
$$

Al-Kharsani and Al-Khal [6] (see also [14]) consider a harmonic generalization of the operator $H_{q, s}$. Let $H_{\mathcal{H}}^{s, \tau}: \mathcal{H} \rightarrow \mathcal{H}$ denote the operator defined by

$$
H_{\mathcal{H}}^{s, \tau} f:=H_{s+1, s} h+\tau \overline{H_{s+1, s} g} \quad(f=h+\bar{g} \in \mathcal{H},|\tau|=1) .
$$

Let $\mathcal{S}_{\mathcal{H}}^{s, \tau}(A, B)$ denote the class of functions $f \in \mathcal{S}_{\mathcal{H}}$ such that

$$
\frac{H_{\mathcal{H}}^{s+1,-\tau} f(z)}{H_{\mathcal{H}}^{s \tau} f(z)} \prec \frac{1+A z}{1+B z} \quad\left(\alpha_{s+2}=2, \beta_{s+1}=1\right) .
$$

Also, by $\mathcal{R}_{\mathcal{H}}^{s, \tau}(A, B)$ we denote the class of functions $f \in \mathcal{S}_{\mathcal{H}}$ such that

$$
\frac{H_{\mathcal{H}}^{s, \tau} f(z)}{z} \prec \frac{1+A z}{1+B z} .
$$

It is clear that

$$
\mathcal{S}_{\mathcal{H}}^{s, \tau}(A, B)=\mathcal{W}_{\mathcal{H}}\left(\varphi_{\tau} ; A, B\right), \quad \mathcal{R}_{\mathcal{H}}^{s, \tau}(A, B)=\mathcal{V}_{\mathcal{H}}\left(\varphi_{\tau} ; A, B\right),
$$

where

$$
\varphi_{\tau}(z)=z F_{s+1, s}(z)+\tau \overline{z F_{S+1, s}(z)} \quad(z \in \mathbb{U}) .
$$

In particular, if we put $s=\beta_{1}=\alpha_{2}=\beta_{2}=1, \alpha_{1}=1+n \in \mathbb{N}, \tau=(-1)^{n}$, then we obtain the classes

$$
\mathcal{S}_{\mathcal{H}}^{n}(A, B):=\mathcal{S}_{\mathcal{H}}^{1,(-1)^{n}}(A, B), \mathcal{R}_{\mathcal{H}}^{n}(A, B):=\mathcal{R}_{\mathcal{H}}^{1,(-1)^{n}}(A, B)
$$

related to the harmonic Ruscheweyh derivatives $\mathcal{D}_{\mathcal{H}}^{n}(f):=H_{\mathcal{H}}^{1, \tau}(f)$ (see [15]). If we put $s=n \in \mathbb{N}_{0}, \tau=(-1)^{n}, \alpha_{k}=2, \beta_{k}=1(k=1,2, \ldots n)$, then we obtain the classes

$$
\mathcal{V}_{\mathcal{H}}^{n}(A, B):=\mathcal{S}_{\mathcal{H}}^{n,(-1)^{n}}(A, B), \mathcal{W}_{\mathcal{H}}^{n}(A, B):=\mathcal{R}_{\mathcal{H}}^{n,(-1)^{n}}(A, B)
$$

related to the harmonic Sălăgean operator $J_{\mathcal{H}}^{n}(f):=H_{\mathcal{H}}^{n, \tau}(f)($ see $[7,11,17,37])$. The classes $\mathcal{S}_{\mathcal{H}}(A, B):=\mathcal{S}_{\mathcal{H}}^{0}(A, B)=\mathcal{V}_{\mathcal{H}}^{0}(A, B), \mathcal{K}_{\mathcal{H}}(A, B):=\mathcal{S}_{\mathcal{H}}^{1}(A, B)=$ $\mathcal{V}_{\mathcal{H}}^{1}(A, B)$, and $\mathcal{R}_{\mathcal{H}}(A, B):=\mathcal{R}_{\mathcal{H}}^{1}(A, B)=\mathcal{W}_{\mathcal{H}}^{1}(A, B)$ are defined in [12] (see also [17]).

The classes $\mathcal{S}_{\mathcal{H}}^{n}(A, B)$ and $\mathcal{R}_{\mathcal{H}}^{n}(A, B)$ are related to harmonic starlike functions, harmonic convex functions and harmonic Janowski functions. The classes $\mathcal{S}_{\mathcal{H}}(\alpha):=$ $\mathcal{S}_{\mathcal{H}}^{0}(2 \alpha-1,1)$ and $\mathcal{K}_{\mathcal{H}}(\alpha):=\mathcal{S}_{\mathcal{H}}^{1}(2 \alpha-1,1)$ are investigated by Jahangiri [19] (see also [3,30]). They are the classes of starlike and convex functions of order $\alpha$, respectively. The classes $N_{\mathcal{H}}(\alpha):=\mathcal{R}_{\mathcal{H}}^{1}(2 \alpha-1,1)$ and $R_{\mathcal{H}}(\alpha):=\mathcal{R}_{\mathcal{H}}^{2}(2 \alpha-1,1)$ are studied in [1] (see also [23]). Finally, the classes $\mathcal{S}_{\mathcal{H}}^{*}:=\mathcal{S}_{\mathcal{H}}(0)$ and $\mathcal{K}_{\mathcal{H}}:=\mathcal{K}_{\mathcal{H}}(0)$ are the classes of functions which are starlike and convex in $\mathbb{U}(r)$, respectively, for 
all $r \in(0,1\rangle$ (see [2]). We should notice, that the classes $\mathcal{S}(A, B):=\mathcal{S}_{\mathcal{H}}(A, B) \cap \mathcal{A}$ and $\mathcal{R}(A, B):=\mathcal{R}_{\mathcal{H}}(A, B) \cap \mathcal{A}$ are introduced by Janowski [22].

The class $\mathcal{W}_{\mathcal{H}}(\varphi ; A, B)$ generalize also classes of starlike functions of complex order. The class $\mathcal{C S}_{\mathcal{H}}(\gamma):=\mathcal{S}_{\mathcal{H}}(2 ; 1-2 \gamma, 1) \quad(\gamma \in \mathbb{C} \backslash\{0\})$ was defined by Yalçin and Öztürk [36]. In particular, if we put $\gamma:=\frac{1-\alpha}{1+e^{i \eta}}$, then we obtain the class $\mathcal{R S}_{\mathcal{H}}(\alpha, \eta):=\mathcal{S}_{\mathcal{H}}\left(2 ; \frac{2 \alpha-1+e^{i \eta}}{1+e^{i \eta}}, 1\right)$ studied by Yalçin et al. [37]. It is the class of functions $f \in \mathcal{H}_{0}$ such that

$$
\operatorname{Re}\left\{\left(1+e^{i \eta}\right) \frac{D_{\mathcal{H}} f(z)}{f(z)}-e^{i \eta}\right\}>\alpha \quad(z \in \mathbb{U}, \eta \in \mathbb{R}) .
$$

Thus, using the obtained results to the classes defined above we can obtain new and also well-known results (see for example [4-16,19-23,27-37]).

Acknowledgements The work is supported by the Centre for Innovation and Transfer of Natural Sciences and Engineering Knowledge, University of Rzeszów.

Open Access This article is distributed under the terms of the Creative Commons Attribution 4.0 International License (http://creativecommons.org/licenses/by/4.0/), which permits unrestricted use, distribution, and reproduction in any medium, provided you give appropriate credit to the original author(s) and the source, provide a link to the Creative Commons license, and indicate if changes were made.

\section{References}

1. Ahuja, O.: Connections between various subclasses of planar harmonic mappings involving hypergeometric functions. Appl. Math. Comput. 198, 305-316 (2008)

2. Ahuja, O.P.: Planar Harmonic Univalent and Related Mappings. J. Inequal. Pure Appl. Math. 6, Art. $122(2005)$

3. Ahuja, O.P., Jahangiri, J.M.: Certain multipliers of univalent harmonic functions. Appl. Math. Lett. 18, 1319-1324 (2005)

4. Al-Hawary, T., Frasin, B.A., Darus, M.: Fekete-Szegö problem for certain classes of analytic functions of complex order defined by the Dziok-Srivastava operator. Acta Math. Vietnam. 39, 185-192 (2014)

5. Al-Khal, R.A.: Goodman-Rřnning-type harmonic univalent functions based on Dziok-Srivastava operator. Appl. Math. Sci. 5, 573-584 (2011)

6. Al-Kharsani, H.A., Al-Khal, R.A.: Univalent harmonic functions. JIPAM. J. Inequal. Pure Appl. Math. 8(2), 8 Article 59 (2007)

7. Al-Shaqsi, K., Darus, M., Fadipe-Joseph, O.A.: A new subclass of Salagean-type harmonic univalent functions. Abstr. Appl. Anal. Art. ID 821531, 12 pp (2010)

8. Aouf, M.K., Srivastava, H.M.: Some families of starlike functions with negative coefficients. J. Math. Anal. Appl. 203, 762-790 (1996)

9. Clunie, J., Sheil Small, T.: Harmonic univalent functions. Ann. Acad. Sci. Fenn. Ser. A I Math. 9, 3-25 (1984)

10. Darus, M., Al-Shaqsi, K.: On certain subclass of harmonic univalent functions. J. Anal. Appl. 6, 17-28 (2008)

11. Dixit, K.K., Porwal, S.: A subclass of harmonic univalent functions with positive coefficients. Tamkang J. Math. 41, 261-269 (2010)

12. Dziok, J.: On Janowski harmonic functions. J. Appl. Anal. 21(2), 99-107 (2015)

13. Dziok, J.: Classes of harmonic functions associated with Ruscheweyh derivatives. Rev. R. Acad. Cienc. Exactas Fís. Nat. Ser. A Math. RACSAM. https://doi.org/10.1007/s13398-018-0542-8 (2018)

14. Dziok, J.: Harmonic function with correlated coefficients. Acta Math. Sci. Ser. B Engl. Ed. (to appear)

15. Dziok, J., Darus, M., Sokół, J., Bulboaca, T.: Generalizations of starlike harmonic functions. C. R. Math. Acad. Sci. Paris 354, 13-18 (2016) 
16. Dziok, J., Srivastava, H.M.: Certain subclasses of analytic functions associated with the generalized hypergeometric function. Integral Transf. Spec. Funct. 14, 7-18 (2003)

17. Dziok, J., Jahangiri, J.M., Silverman, H.: Harmonic functions with varying coefficients. J. Inequal. Appl. 2016, 139 (2016)

18. Hallenbeck, D.J., MacGregor, T.H.: Linear Problems and Convexity Techniques in Geometric Function Theory. Pitman Advanced Publishing Program, Boston, Pitman (1984)

19. Jahangiri, J.M.: Harmonic functions starlike in the unit disk. J. Math. Anal. Appl. 235, 470-477 (1999)

20. Jahangiri, J.M., Kim, Y.C., Srivastava, H.M.: Construction of a certain class of harmonic close-toconvex functions associated with the Alexander integral transform. Integral Transf. Spec. Funct. 14, 237-242 (2003)

21. Jahangiri, J.M., Silverman, H.: Harmonic univalent functions with varying arguments. Int. J. Appl. Math. 8, 267-275 (2002)

22. Janowski, W.: Some extremal problems for certain families of analytic functions I. Ann. Polon. Math. 28, 297-326 (1973)

23. Karpuzoullari, S.Y., Öztürk, M., Yamankaradeniz, M.: A subclass of harmonic univalent functions with negative coefficients. Appl. Math. Comput. 142(2-3), 469-476 (2003)

24. Krein, M., Milman, D.: On the extreme points of regularly convex sets. Stud. Math. 9, 133-138 (1940)

25. Lewy, H.: On the non-vanishing of the Jacobian in certain one-to-one mappings. Bull. Am. Math. Soc. 42, 689-692 (1936)

26. Montel, P.: Sur les families de functions analytiques qui admettent des valeurs exceptionelles dans un domaine. Ann. Sci. Ecole Norm. Sup. 23, 487-535 (1912)

27. Mostafa, A.O., Aouf, M.G.: Goodman-Rřnning-type multivalent harmonic functions based on DziokSrivastava operator. Southeast Asian Bull. Math. 39, 829-840 (2015)

28. Murugusundaramoorthy, G., Vijaya, K.: Certain classes of harmonic functions associated with DziokSrivastava operator. An. Univ. Oradea Fasc. Mat. 17, 75-86 (2010)

29. Omar, R., Halim, S.A.: Multivalent harmonic functions defined by Dziok-Srivastava operator. Bull. Malays. Math. Sci. Soc. 2(35), 601-610 (2012)

30. Öztürk, M., Yalçin, S., Yamankaradeniz, M.: Convex subclass of harmonic starlike functions. Appl. Math. Comput. 154, 449-459 (2004)

31. Ruscheweyh, S.: Convolutions in geometric function theory. Sem. Math. Sup. 83, Les Presses del'Université de Montréal (1982)

32. Sălăgean, G.S.: Subclasses of univalent functions. Lecture Notes in Mathematics. Springer, New York, vol. 1013, pp. 362-372 (1983)

33. Sheil-Small, T.: Constants for planar harmonic mappings. J. Lond. Math. Soc. 2(42), 237-248 (1990)

34. Silverman, H.: Harmonic univalent functions with negative coefficients. J. Math. Anal. Appl. 220, 283-289 (1998)

35. Xu, Q.-H., Xiao, H.-G., Srivastava, H.M.: Some applications of differential subordination and the Dziok-Srivastava convolution operator. Appl. Math. Comput. 230, 496-508 (2014)

36. Yalçin, S., Öztürk, M.: Harmonic functions starlike of the complex order. Mat. Vesnik 58, 7-11 (2006)

37. Yalçin, S., Öztürk, M., Yamankaradeniz, M.: On the subclass of Salagean-type harmonic univalent functions. JIPAM. J. Inequal. Pure Appl. Math. 8, 9 Article 54 (2007)

Publisher's Note Springer Nature remains neutral with regard to jurisdictional claims in published maps and institutional affiliations. 